\title{
Workshop como uma metodologia para o ensino- aprendizagem de ciências: um estudo de caso com o grupo Bioinorganic Chemistry Environment and Medicine (BIOMET)
}

\author{
Hector Aguilar Vitorino ${ }^{1}$ (D), Roxana Yesenia Pastrana Alta ${ }^{1}$ (D) \& Priscila \\ Ortega $^{2}$ (1)
}

(1) Universidad Nacional de Ingeniería, Facultad de Ciéncias, Av. Túpac Amaru 210, Rimac, Lima, Perú. E-mail: havitorino@gmail.com, rpastranaa@uni.edu.pe

(2) Universidade de São Paulo, Instituto de Biociências, Rua do Matão 321 - travessa 14, Cidade Universitária 05508-090, São Paulo, Brasil. E-mail: priortega218@gmail.com

Vitorino H.A., Alta R.Y.P. \& Ortega P. (2021) Workshop como uma metodologia para o ensinoaprendizagem de ciências: um estudo de caso com o grupo Bioinorganic Chemistry Environment and Medicine (BIOMET). Pesquisa e Ensino em Ciências Exatas e da Natureza, 5: e1699.

http://dx.doi.org/10.29215/pecen.v5i0.1699

Editor acadêmico: Hugo da Silva Florentino. Recebido: 01 dezembro 2020. Aceito: 22 abril 2021. Publicado: 30 abril 2021.

Resumo: A busca por metodologias educacionais, como os workshops, é constante para a melhoria do processo de ensino-aprendizagem. Dessa maneira, o objetivo do estudo foi analisar o workshop BIOMET 2017 como um evento de difusão científica e ferramenta metodológica para o aprendizado significativo dos participantes. O evento ocorreu em 3 dias, sendo o primeiro dia teórico (palestras sobre bioindicadores marinhos), o segundo prático (minicurso) e o terceiro dia de análise e discussão de dados. Ao final do terceiro dia, um formulário de satisfação foi dado para cada participante para avaliação do evento. De maneira geral, o evento apresentou grande participação masculina de estudantes de graduação, das áreas de Química e Biologia, com pouca experiência em laboratório, e classificado como "bom ou muito bom" pelos participantes. O minicurso avaliou estatisticamente a morte de artêmias, ao longo do tempo, em crescentes concentrações de cloreto de cádmio. A morte de artêmias ocorreu a partir de $10 \mathrm{mmol} \mathrm{L}^{-1}$, após 1 hora de experimento, os quais contrastaram com a literatura, possivelmente devido às dificuldades relacionadas a execução do experimento. Com esse intuito, eventos como BIOMET 2017 são importantes ferramentas de aprimoração, aproximando o conteúdo teórico da prática laboratorial, desenvolvendo profissionais mais críticos e reflexivos.

Palavras chave: Educação, minicurso, artêmias, contaminantes.

Workshop as a methodology for science teaching-learning: a case study with the Bioinorganic Chemistry Environment and Medicine group (BIOMET)

Abstract: The search for educational methodologies, such as workshops, is constant to improve the teaching-learning process. Thus, the objective of the study was to analyze the BIOMET 2017 workshop as a scientific dissemination event and methodological tool for the participants' significant learning. The event took place in 3 days, with the first theoretical day (lectures on marine bioindicators), the second practical (mini-course) and the third day of data analysis and discussion. At the end of the third day, a satisfaction form was given to each participant to evaluate the event. In general, the event featured a large male participation of undergraduate students, from the areas of Chemistry and Biology, with little experience in the laboratory, and classified as "good or very good" by the participants. The mini-course statistically evaluated the death of fairy shrimp, over time, in increasing concentrations of cadmium chloride. The death of fairy shrimp occurred after $10 \mathrm{mmol} \mathrm{L}^{-1}$, after $1 \mathrm{~h}$ of 
the experiment, which contrasted with the literature, possibly due to the difficulties related to the execution of the experiment. To that end, events as BIOMET 2017 are important tools for improvement, bringing theoretical content closer to laboratory practice, developing more critical and reflective professionals.

Key words: Education, mini-course, fairy shrimp, contaminants.

\section{Introdução}

As transformações sociais têm colocado em discussão os aspectos educacionais e a formação de profissionais. A situação do ensino, principalmente de Ciências, é uma preocupação mundial (Krasilchik 2009). Dados, principalmente de exames internacionais, revelam que o aprendizado do aluno de vários países está abaixo do esperado e está distante das metas apontadas para o ensino, como transcender a memorização de informações desconexas e irrelevantes (Krasilchik 2009). Estudos revelam que os alunos, em geral, ouvem falar sobre ciência, contudo, não aprendem a pensar cientificamente (Alberts 2009). Nesse contexto, o ensino de Ciências precisa ser melhorado, para que este ocupe lugar essencial na educação dos estudantes (Krasilchik 2009). Reforçando essa ideia inicial, estudos indicam que o objetivo do ensino de Ciências não é apenas formar uma pequena parcela de cientistas, mas sim, alfabetizar a população cientificamente, para que esta se preocupe com os desafios globais que a humanidade enfrenta, e assim tomar decisões sábias baseadas na ciência (Wieman 2007).

Atualmente, acredita-se que a educação precisa favorecer o desenvolvimento sistêmico e ecológico do homem, para um melhor desempenho no seu âmbito de trabalho (Cotta et al. 2012). Além disso, a contextualização dos fatos, assim como a maior participação dos alunos no seu próprio processo de ensino-aprendizagem, são aspectos importantes para a melhoria da educação (Festas 2015). Historicamente, os discentes apresentam uma formação conservadora, baseada em metodologias de ensino tradicionais, sendo estas fragmentadas e reducionistas (Cyrino \& Toralles-Pereira 2004; Cotta et al. 2012).

As práticas tradicionais de ensino, por muitas vezes, restringem o processo ensinoaprendizagem, sendo este voltado para a reprodução do conhecimento, no qual o educador detém o poder do conhecimento, assumindo a postura de transmissor de conteúdos e formulador de questões (Cyrino \& Toralles-Pereira 2004; Cotta et al. 2012). Por outro lado, os educandos apresentam-se como indivíduos que necessitam apenas dar as respostas corretas, absorver e repetir conteúdos, sem a criticidade necessária para a reflexão dos conteúdos, sendo estes memorizados (Cyrino \& Toralles-Pereira 2004; Cotta et al. 2012). Esta prática, conhecida como concepção "bancária", se restringe ao ato de depositar, transferir e reproduzir valores e conhecimentos, sendo estes indivíduos passivos e acríticos, representando um dos principais obstáculos para o pleno desenvolvimento do discente (Freire 1987). Atualmente, a educação passa por um processo de transformação. Metodologias conservadoras, nas quais o aluno é passivo dentro do ambiente educacional, não são tão eficazes durante o processo de ensinoaprendizagem (Freire 1987). Assim, torna-se cada vez mais importante a inovação metodológica para garantir maior aprendizagem dos alunos e significância dos conteúdos (Krasilchik 2009; Valderrama et al. 2016). Dessa maneira, os alunos precisam estar inseridos na estrutura educacional, os quais são o centro do aprendizado. Metodologias práticas, na maioria das vezes grupais, as quais conduzem à resolução de situações-problema, são as novas perspectivas para uma educação mais inclusiva e com maior significância no aprendizado (Krasilchik 2009).

Assim, novas práticas pedagógicas e metodológicas começaram a surgir, com o objetivo de tornar o aprendizado mais problematizador e significativo. Segundo estudos (Borochovicius \& Tortella 2014; Wieman \& Gilbert 2015; Fagundes 2016), para que ocorra o aprendizado significativo, a educação precisa ser transformacional, em que o aprendiz consegue relacionar os conceitos (sejam novos ou remodelados), às suas experiências prévias e vivências, permitindo a formulação de problemas estimuladores, desafiadores e que incentivem novas aprendizagens (Borochovicius \& Tortella 2014). Dessa forma, aprender significativamente significa relacionar, de maneira substantiva, a nova informação com uma estrutura de conhecimento específica que 
faz parte integrante da estrutura cognitiva prévia do aprendiz. Assim, o conceito de aprendizagem significativa está relacionado com o processo de assimilação substantiva do que se aprende, o qual interage com um conhecimento prévio, podendo este ser reformulado, ou remodelado (Valadares 2011). Nesse mesmo sentido, Madruga (1996) mostra que metodologias problematizadoras são significativas, valorizando o aprender a aprender, ressaltando a necessidade de conceber a educação como prática de liberdade, em oposição a uma educação como prática de dominação (Freire 1987).

Nesse sentido, a prática de metodologias diferenciadas e que gerem questões a serem debatidas e discutidas são importantes formas de aprendizado significativo. Dentre essas práticas, encontram-se os workshops e minicursos. De maneira geral, os workshops são encontros nos quais um grupo de pessoas/estudantes são estimulados a resolverem questões problematizadoras, com o intuito de adquirir novos conhecimentos ou aprofundá-los (Ørngreen \& Levinsen 2017). Dessa forma, os workshops podem ser utilizados como métodos diferenciados para um aprendizado ou aprofundamento do conhecimento de maneira significativa. Além disso, os workshops também são importantes para o ensino-aprendizagem de Ciências, pois é um espaço para a construção da argumentação, possibilitando a criação de uma cultura científica. Sendo assim, podem ser considerados como ambientes de aprendizagem efetivamente argumentativos, em que os participantes podem exercitar as habilidades argumentativas e aprender conteúdos de ciência e sobre ciência (Scarpa 2015).

BIOMET (Bioinorganic Chemistry Environment and Medicine) é um grupo de pesquisa da Facultad de Ciencias da Universidad Nacional de Ingenieria (UNI - Lima, Perú), o qual realiza, uma vez ao ano, eventos abertos ao público em geral, promovendo a discussão científica com relação à aplicação da Química Bioinorgânica no meio ambiente. O evento BIOMET iniciou em dezembro de 2014, primeiramente como um encontro de estudantes. Esse evento apresentou, de maneira geral, as linhas de pesquisa dos estudantes e como as instituições universitárias desenvolviam seus projetos de pesquisa. Em 2015, BIOMET iniciou com um workshop sobre análises de dados e, a partir de 2016, passou a realizar práticas laboratoriais, inclusive com a publicação de um artigo científico.

Este evento ocorre uma vez ao ano, na UNI, sempre no mês de dezembro, com o intuito de divulgar as pesquisas científicas que ocorrem dentro e fora do país. Além disso, o workshop visa a participação dos alunos em atividades práticas laboratoriais, para que este conheça a rotina de um cientista. Devido aos problemas ambientais enfrentados recentemente, como a poluição da água dos rios e dos oceanos, com o aumento do descarte de resíduos sólidos, metais tóxicos e plástico no meio ambiente, BIOMET esteve à frente, nesses últimos anos, de palestras e práticas laboratoriais voltadas para o estudo, e possível mitigação e conservação do ambiente natural.

Neste contexto, os objetivos do presente artigo são: 1. analisar a possibilidade de um workshop (BIOMET 2017) como uma forma de difusão científica e aprendizagem; e 2. refletir sobre como o minicurso desenvolvido no workshop pode contribuir na formação inicial de estudantes que frequentaram poucas aulas experimentais. Para isso, o evento apresentou palestras de profissionais da área, e um minicurso teórico/prático com posterior análise dos dados obtidos. No final do evento, um formulário de satisfação foi respondido pelos participantes com o intuito de compreender como BIOMET 2017 contribuiu para a difusão científica e aprendizagem dos participantes.

\section{Material e Métodos}

\section{O evento}

O evento detalhado no presente artigo apresentou-se como uma ferramenta metodológica para um aprendizado significativo dos alunos, pois contava com parte teórica e experimental conectadas, na qual os alunos poderiam relacionar a teoria com a prática. As palestras realizadas na área ambiental e os resultados encontrados pelos cientistas formaram a parte teórica. $\mathrm{O}$ minicurso na temática ambiental e a discussão dos dados encontrados representaram a parte prática do evento, unindo-se com a teoria apresentada anteriormente. Durante o minicurso, 
aspectos relacionados ao método científico como a observação do experimento em si e dos resultados, o levantamento de hipóteses, a tentativa de explicação dessas e o processo argumentativo para a discussão e conclusão dos dados, também foram importantes para o aprendizado significativo.

O workshop BIOMET 2017 apresentou, como tema principal, pesquisas relacionadas à área de ecotoxicologia, com ênfase em bioindicadores marinhos. A duração de cada palestra foi em torno de 30 a 45 minutos, com 15 minutos para perguntas e discussão do tema apresentado.

Como acontece todos os anos, a publicidade do evento BIOMET 2017 ocorreu via web (https://www.bicsociety.org/biomet2017) e redes sociais (Facebook); além disso, pôsteres, folderes e anúncios foram distribuídos e fixados na UNI e em outras universidades e institutos de ensino peruanos, realizando assim o marketing do evento.

BIOMET 2017 foi realizado nos dias 18, 19 e 20 de dezembro de 2017. O primeiro dia contou com palestras relacionadas ao tema principal do evento (bioindicadores marinhos e contaminação ambiental). No primeiro dia, o evento apresentou 4 palestras, as quais estavam organizadas em: (1) estresse oxidativo; (2) quantificação dos fatores de estresse oxidativos em bioindicadores marinhos; (3) toxicidade em artêmias; e (4) toxicidade em células de caranguejos. Os palestrantes eram bacharéis e doutores na área de Química e Biologia, sendo professores pesquisadores com experiência em Bioinorgânica e Ecotoxicologia. No segundo dia de evento foi realizado o minicurso, com a exposição de Artemia salina ao contaminante cádmio durante 5 horas. O minicurso apresentou uma parte teórica introdutória de 15 minutos e, em seguida, o experimento foi detalhado com os participantes do minicurso, com o objetivo de sanar possíveis dúvidas quanto à sua execução. O terceiro dia, por sua vez, apresentou somente os resultados e discussão do minicurso realizado no dia anterior, com introdução à programas para a confecção de gráficos, como o Origin e GraphPrism, além do uso de programas para a organização de referências (Mendeley) e para a análise de dados (Sigma Stat). No final do evento, foram distribuídos formulários de satisfação para os participantes (MS1 e MS2), para obter dados sobre a importância e relevância do evento.

\section{Minicurso}

Um dia antes da realização do minicurso, as artêmias (Artemia salina) encapsuladas (ovos) foram colocadas para eclosão em um béquer contendo água do mar filtrada (35\%), segundo estudos realizados anteriormente (Lima et al. 2009). Durante a eclosão (24 horas), as artêmias foram mantidas sob iluminação e aeração constantes, a $25 \pm 3^{\circ}$ C. No dia 19 de dezembro de 2017 foi realizado o minicurso no Laboratório de Química da UNI. Em placas de cultura de 6 poços (KASVI), 24 nauplios de artêmias foram colocados em cada poço, contendo água do mar filtrada $(35 \%$ ) com diferentes concentrações de contaminante (cloreto de cádmio $\mathrm{CdCl}_{2}$ ). A primeira placa foi destinada como controle (24 artêmias + água do mar filtrada), enquanto que as duas placas seguintes foram as experimentais (24 artêmias + água do mar filtrada $+\mathrm{CdCl}_{2}$ ), obtendo, assim, um total de 3 placas. Os contaminantes foram adicionados em concentrações crescentes de 2 a $24 \mathrm{mmol} \mathrm{L}^{-1}\left(2,4,6,8,10,12,14,16,18,20,22\right.$ e $\left.24 \mathrm{mmol} \mathrm{L}^{-1}\right)$, sendo a placa experimental 1 composta pelas concentrações de 2 a $12 \mathrm{mmol} \mathrm{L}^{-1}$, e a placa experimental 2 composta pelas concentrações de 14 a $24 \mathrm{mmol} \mathrm{L}^{-1}$. O experimento foi realizado durante 5 horas, onde, a cada 1 hora, as placas controle, experimental 1 e 2 eram visualizadas, com a contagem das artêmias mortas (organismos que não se moviam).

Cada participante (total de 20 cursistas) realizou o experimento completo (controle e experimento 1 e 2), constituindo, assim 20 réplicas. Foram utilizadas $n=24$ artêmias para $o$ grupo controle e para o grupo experimental, com $n=8640$ artêmias utilizadas durante todo $o$ minicurso.

\section{Avaliação}

A avaliação do workshop foi realizada no primeiro e no último dia do evento, no qual foram distribuídos aos participantes dois questionários, anônimos e não obrigatórios, com 
perguntas de múltipla escolha e dissertativas. Antes de serem respondidos, os formulários foram lidos e explicados aos participantes do evento, para que estes soubessem o seu conteúdo. A divulgação dos resultados dos formulários também foi autorizada pelos participantes do evento. O questionário 1 foi respondido no primeiro dia do evento, e apresentou um total de 12 perguntas via formulário web (google docs forms - MS1), as quais estavam divididas em questões pessoais (idade dos participantes, sexo, presença de deficiências físicas, como soube do workshop), questões profissionais (experiência em laboratório; conhecimento em processamento de dados experimentais, análise estatística e referências; experiência com manejo de animais) e questões envolvendo a temática principal do evento (realização de trabalhos de pesquisa ou estudos com bioindicadores marinhos, interesse na área de bioindicadores, utilização de protocolos com bioindicadores para determinar o grau de poluição de um ambiente). Logo após o envio das respostas pelos participantes, o questionário foi avaliado para a escolha dos participantes do minicurso. Levou-se em consideração para a participação do minicurso alunos que apresentavam baixa frequência de aulas de laboratório em suas universidades (aulas laboratoriais uma vez na semana, com realização de um único experimento/aula), e pouca experiência com bioindicadores, com o intuito de apresentar metodologias laboratoriais, e aprendizados/experiências científicas diferentes aos participantes do minicurso.

$\mathrm{O}$ questionário 2 foi respondido pelos participantes no último dia de evento, e apresentou um total de 9 perguntas, as quais também constavam com questões pessoais (conhecimento do curso de Química na UNI, temas para a realização de novos workshops, presença de workshops em suas universidades de destino, novos temas para capacitação em laboratório, e grau de satisfação com o workshop realizado) e questões educacionais (frequência e experimentos realizados em aulas de laboratório de suas universidades de destino, utilização de protocolos experimentais, presença de clubes de Ciência ou de algum outro tipo de difusão científica). Esse questionário foi entregue aos administradores do evento logo após o término deste, o qual foi avaliado e utilizado para determinar o grau de satisfação dos participantes (MS 2).

\section{Análise estatística}

Os dados dos formulários (via google docs e de satisfação) foram compilados e apresentados em forma de tendência, utilizando o programa OriginGraphPro 2016. Os resultados experimentais obtidos no minicurso foram apresentados como média \pm desvio padrão. Kruskal-Wallis Two-way Analysis of Variance on Ranks (ANOVA) foi utilizado para a análise dos dados de porcentagem de morte das artêmias em relação ao tempo de exposição e às diferentes concentrações de cloreto de cádmio em comparação com os dados controle. Os dados de normalidade e homogeneidade foram checados pelo teste Holm-Sidak, realizado pelo programa estatístico Sigma Stat para Windows 3.10. O grau de significância utilizado foi de 5\% utilizando o teste Tukey post hoc (Rohlf \& Sokal 1969).

\section{Resultados}

\section{O evento}

O evento BIOMET 2017 aportou 90 inscritos, de universidades estritamente peruanas, os quais pertenciam majoritariamente à UNI, com $24.85 \%$ de participantes, e à Universidad Nacional Mayor de San Marcos, com 19.2\% de alunos participantes. As duas universidades juntas apresentaram $44.05 \%$ dos participantes do evento (Figura 1A). Pode-se destacar também a grande participação ( $14.69 \%$ do público presente) de alunos pertencentes à Universidad Cayetano Heredia. Em porcentagens inferiores, mas não menos importantes, encontram-se também os alunos pertencentes às Universidad Científica del Sur, com $11.33 \%$ de participantes no evento, e a Universidad Nacional Federico Villareal, com 10.16\% de participantes. Com $4.52 \%$ de participação, estão os alunos da Universidad Nacional del Callao, e $15.25 \%$ dos participantes pertenceram a outras universidades e/ou institutos da cidade de Lima. Dentre os inscritos, 
$48.31 \%$ participaram apenas das palestras BIOMET 2017, enquanto que $22.21 \%$ participaram do minicurso e $29.48 \%$ participaram tanto do minicurso quanto das palestras oferecidas (Figura 1B).
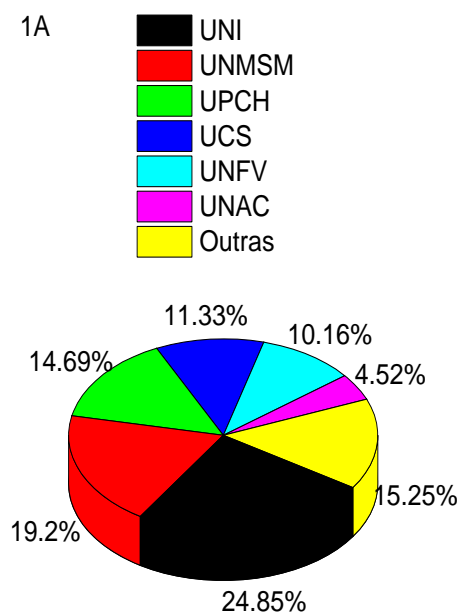

$1 \mathrm{~B}$

Minicurso

Minicurso e palestras

Apenas palestras

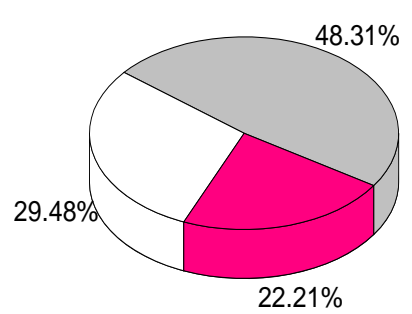

Figura 1. Workshop BIOMET: A. Porcentagem (\%) de participantes do evento BIOMET 2017 e a universidade em que estudam; B. Porcentagem (\%) de participação nas diferentes etapas do workshop. Legenda: UNI = Universidad Nacional de Ingenieria; UNMSM = Universidad Nacional Mayor de San Marcos; UPCH = Universidad Peruana Cayetano Heredia; UCS = Universidad Científica del Sur; UNFV = Universidad Nacional Federico Villareal; UNAC = Universidad Nacional de Callao.

Dentre os participantes, a maioria cursava as especialidades de Química e Biologia (Figura 2). O curso de Química apresentou a maior porcentagem de participantes no evento, com $37.77 \%$ do total de inscritos, enquanto que o curso de Biologia foi o segundo maior em porcentagem de participação no BIOMET, com $22.22 \%$ do total de inscritos. Os cursos de Engenharia Ambiental, Bioquímica, Farmácia e Engenharia Química apresentaram as menores porcentagens de participantes, com $11.10 \%, 6.67 \%, 4.45 \%$ e $4.45 \%$, respectivamente. Outros cursos como Matemática e Estatística, Pedagogia, Física, Medicina e Saúde Pública também contaram com participantes no evento totalizando $13.33 \%$.

Com relação aos participantes do evento, $52 \%$ eram do sexo masculino e $48 \%$ do sexo feminino, independentemente do curso que realizavam ou da universidade que frequentavam (Figura 3A). Do total de participantes, $68 \%$ eram estudantes de graduação, $10 \%$ estudantes de pós-graduação e $6.67 \%$ professores de diversas instituições de ensino. Vale ressaltar a baixa participação de pesquisadores no evento; $1.11 \%$ dos participantes estavam relacionados com alguma linha de pesquisa na área de ecotoxicologia, ou faziam parte de algum laboratório que desenvolviam projetos na área de Ciências (Figura 3B). Dentre os estudantes de graduação, 50\% eram do sexo feminino e $50 \%$ eram do sexo masculino, enquanto que dentre os estudantes de pós-graduação, $40 \%$ eram do sexo feminino e $60 \%$ do sexo masculino; por sua vez, dentre o grupo dos professores e pesquisadores, $90 \%$ eram do sexo masculino e $10 \%$ do sexo feminino (dados não mostrados nos gráficos). A faixa etária dos participantes variou de 18 anos (mínimo), principalmente para alunos de graduação, a 48 anos (máximo), para professores. A média etária em relação ao grau de escolaridade/ocupação foi de: 20.5 anos para estudantes de graduação, 26.5 anos para estudantes de pós-graduação (mestrado e doutorado), 31.5 anos para pesquisadores e 39 anos para professores (Figura 3C). Vale ressaltar que o evento contou com a participação de um público relativamente jovem, em sua maioria alunos de graduação dos ciclos iniciais. 


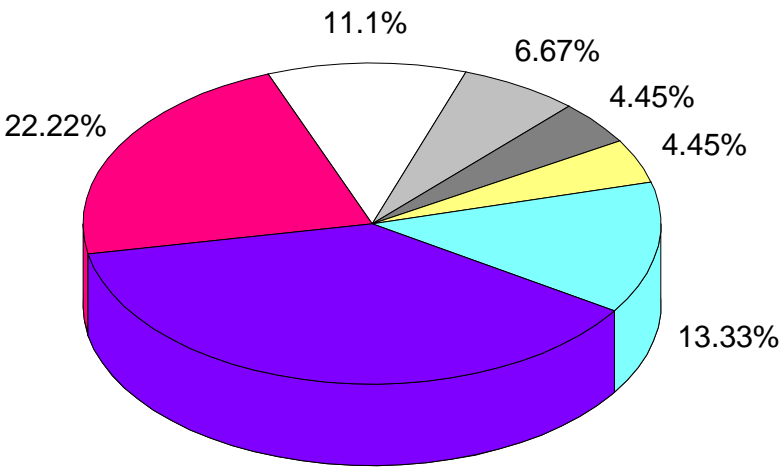

Química

Biologia

Engenharia Ambiental

Bioquímica

Farmácia

Engenharia Química

Outras

$37.77 \%$

Figura 2. Porcentagem (\%) de participantes do evento BIOMET 2017 e os cursos que realizam ou lecionam, independentemente da universidade em que estudam ou trabalham.

$3 \mathrm{~A}$

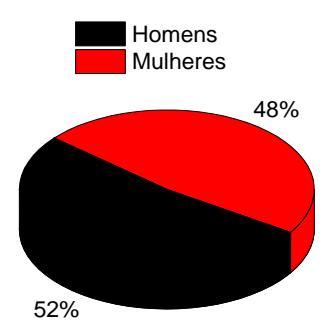

$3 B$

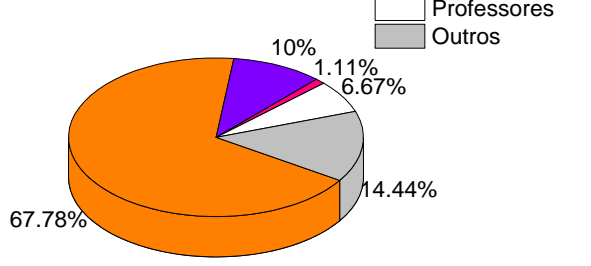

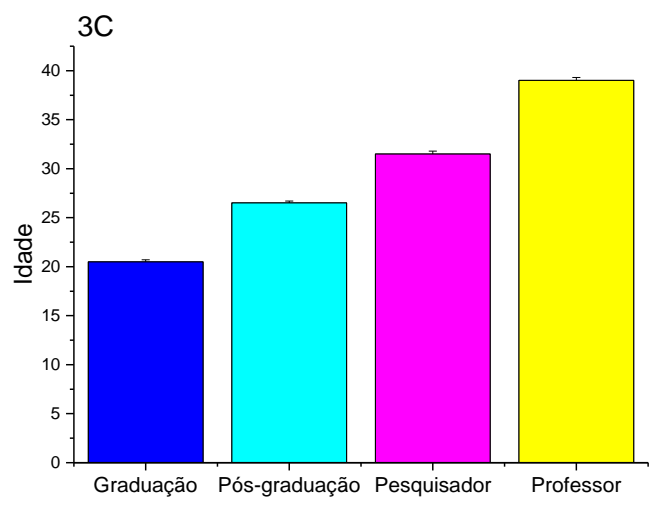

Figura 3. Workshop BIOMET: A. Porcentagem (\%) do total de participantes do sexo masculino e feminino presentes no evento BIOMET 2017; B. Escolaridade/ocupação dos inscritos do evento, independentemente do gênero (masculino ou feminino); G. Faixa etária (média \pm desvio padrão) dos participantes de BIOMET 2017 em relação à escolaridade/ocupação desempenhada.

Com relação à experiência em laboratório (questão apresentada no questionário de avaliação 2), foi perguntado aos participantes a frequência de aulas de laboratório que a universidade/curso oferecia aos seus estudantes. Dentre os inscritos, metade (50\%) dos participantes do evento apresentavam aulas de laboratório uma vez por semana; $10 \%$ frequentavam aulas de laboratório duas vezes na semana; $35 \%$ responderam que as 
universidades/cursos que frequentavam disponibilizavam aulas de laboratório três vezes na semana, enquanto que apenas $5 \%$ dos entrevistados realizavam suas práticas laboratoriais quatro vezes por semana (Figura 4A).

Quando os participantes foram questionados a respeito da frequência de experimentos realizados em suas práticas laboratoriais (questão apresentada no questionário 1), $60 \%$ dos participantes responderam que realizavam apenas um experimento em suas aulas de laboratório; $25 \%$ responderam que realizavam de dois a três experimentos em suas aulas práticas, enquanto que $15 \%$ realizavam de três a quatro experimentos em suas aulas de laboratório (Figura 4B).

$4 \mathrm{~A}$
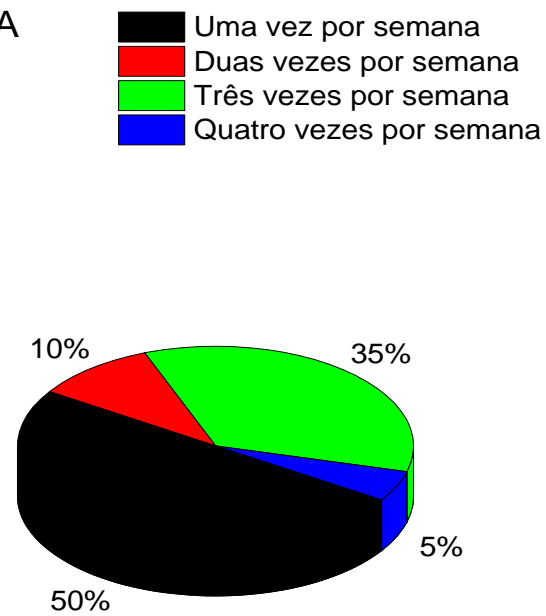

4B
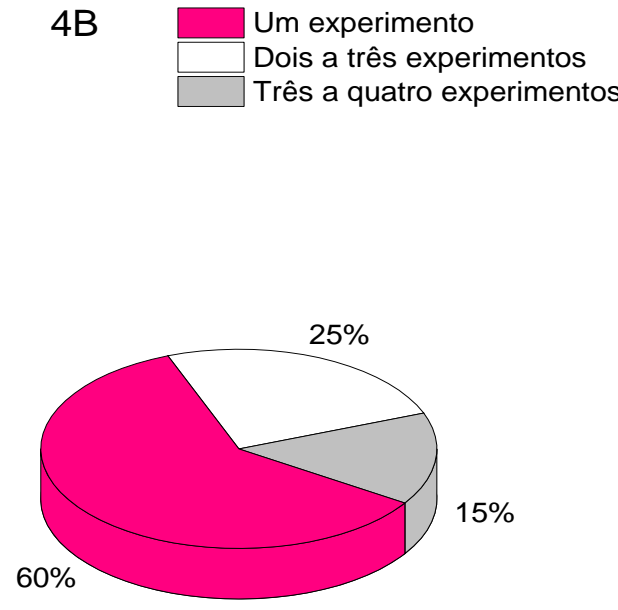

Figura 4. Experiência laboratorial prévia dos alunos inscritos no BIOMET: A. Porcentagem (\%) de aulas de laboratório realizadas nas instituições de ensino dos participantes. As diferentes cores representam as diferentes frequências de aulas de laboratório ofertadas aos estudantes pelas universidades/instituições de ensino/disciplinas que cursavam; B. Porcentagem (\%) de experimentos realizados em aulas de laboratório nas diferentes universidades/instituições de ensino/disciplinas cursadas pelos participantes do evento.

Outro fator importante que necessita ser abordado é a questão da presença de Clubes de Ciência ou algum outro tipo de difusão científica (workshops) realizada pelas instituições de ensino frequentadas pelos participantes. De acordo com os resultados obtidos no formulário de satisfação, mais da metade dos participantes (55\%) desconhecem eventos de difusão científica (Figura 5A). Ressaltando a qualidade do evento BIOMET, 75\% dos participantes consideraram o evento bom ou muito bom (25\%), inclusive superando as expectativas dos participantes (Figura 5B). Quanto ao tema, acredita-se que este deve ser mais aprofundado e com novos experimentos, garantindo novas aprendizagens e capacitações para esses profissionais ou futuros profissionais (Figura 5C).

\section{O minicurso}

O minicurso transcorreu bem, tendo alcançado o seu objetivo inicial de manuseio de equipamentos, aprendizado de metodologias e de técnicas laboratoriais. No entanto, os participantes relataram alguns problemas durante a execução da parte experimental, tais como: (1) manejo das artêmias: os participantes apresentaram dificuldades na transferência das artêmias dos aquários para as placas devido a rápida movimentação dos microcrustáceos; (2) adição das diferentes concentrações de cádmio: os estudantes apresentaram dificuldades para estabilizar o volume final de cada poço das placas, podendo alterar as concentrações propostas no protocolo; (3) contagem das artêmias mortas: os participantes encontraram dificuldades na observação das artêmias com redução na locomoção em relação às artêmias totalmente paralisadas (mortas). 

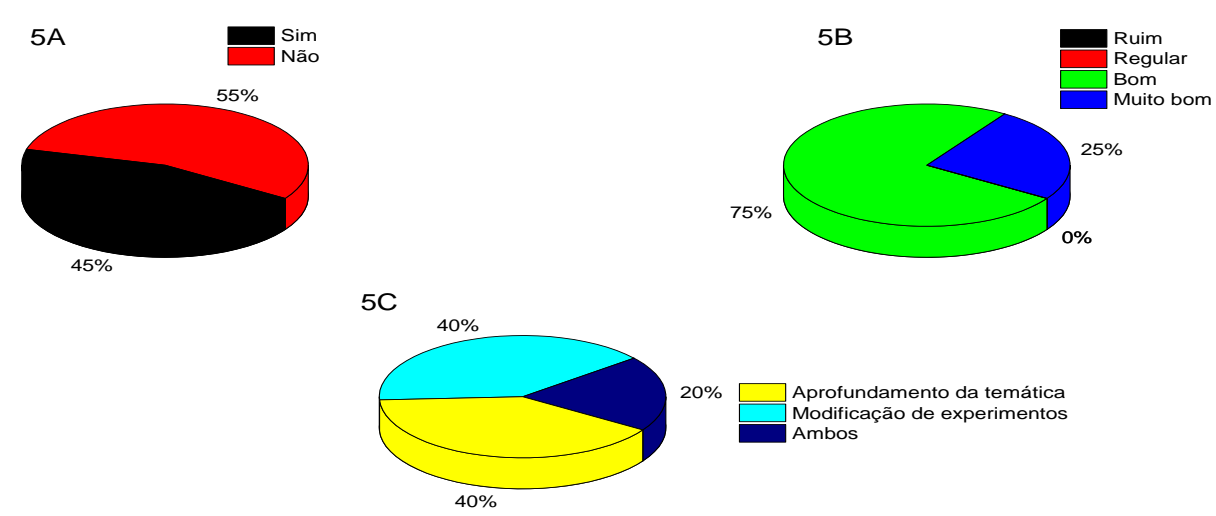

Figura 5. Workshop BIOMET: A. Porcentagem (\%) de participantes com relação aos eventos de difusão científica; B. Porcentagem (\%) da avaliação da qualificação de satisfação dos participantes com relação ao evento BIOMET 2017; C. Porcentagem (\%) de aprofundamento da temática e modificação de experimentos nos minicursos para os próximos workshops BIOMET.

No entanto, após o auxílio inicial dos ministrantes, os participantes do minicurso conseguiram conduzir o experimento proposto sozinhos. Assim, a Figura 6 mostra a porcentagem de artêmias mortas em relação às diferentes concentrações de cloreto de cádmio $\left(\mathrm{CdCl}_{2}\right)$ ao longo de 5 horas de exposição ao metal. Pode-se observar um aumento do percentual de morte de artêmias após 1 hora de exposição ao $\mathrm{Cd}$, principalmente nas concentrações acima de $12 \mathrm{mmol} \mathrm{L}^{-1} \mathrm{de}_{\mathrm{CdCl}_{2}}$. Com relação ao tempo de exposição, a partir de 1 hora de experimento foi significativamente suficiente para ocorrer a morte das artêmias. O aumento da morte dos microcrustáceos ocorreu de acordo com o aumento do tempo de exposição, sendo significativamente maiores nos tempos de 1 a 5 horas de exposição em comparação ao controle. Com relação às concentrações utilizadas, o aumento da morte das artêmias foi significativamente maior nas concentrações de 14 a $24 \mathrm{mmol} \mathrm{L}^{-1}$. No entanto, quando relacionamos o tempo de exposição com as concentrações utilizadas, a morte das artêmias foi significativamente maior nas concentrações de 14 a $24 \mathrm{mmol} \mathrm{L}^{-1}$ de Cd durante 4 a 5 horas de exposição. Contudo, a morte não atingiu a totalidade das artêmias, apresentando $65 \%$ de mortalidade durante 5 horas de experimento.

\section{Discussão}

\section{O evento}

BIOMET 2017 apresentou uma metodologia participativa para alunos da graduação e pósgraduação, fato este que pode ser observado nos resultados do formulário de satisfação. Primeiramente, os dados revelam que BIOMET 2017 alcançou o público local e aqueles que estão nas imediações da universidade que comportou o evento. $O$ maior número de participantes da própria universidade que sediou o evento ocorreu, possivelmente, devido à maior divulgação e publicidade realizada pela instituição de ensino. Cartazes, folders e banners estavam espalhados por todas as imediações da universidade, o que facilitou a sua visualização e conhecimento do evento. Outras universidades, por sua vez, divulgaram o evento apenas por um cartaz na entrada principal da instituição, o que pode ter prejudicado uma divulgação mais efetiva do evento. Além disso, os inscritos apresentaram um perfil voltado para as áreas de Ciências Biológicas e Ambiental, possivelmente devido ao conteúdo das apresentações e do minicurso ministrado no BIOMET 2017, os quais estavam voltados para as áreas de Química, Biologia e Meio ambiente. Dessa forma, pode-se perceber que as universidades que apresentam cursos nas áreas das Ciências, como é o caso da UNI, Universidad Nacional Mayor San Marcos, Universidad Cayetano Heredia e Universidad Científica del Sur, foram as principais instituições participantes do evento. 


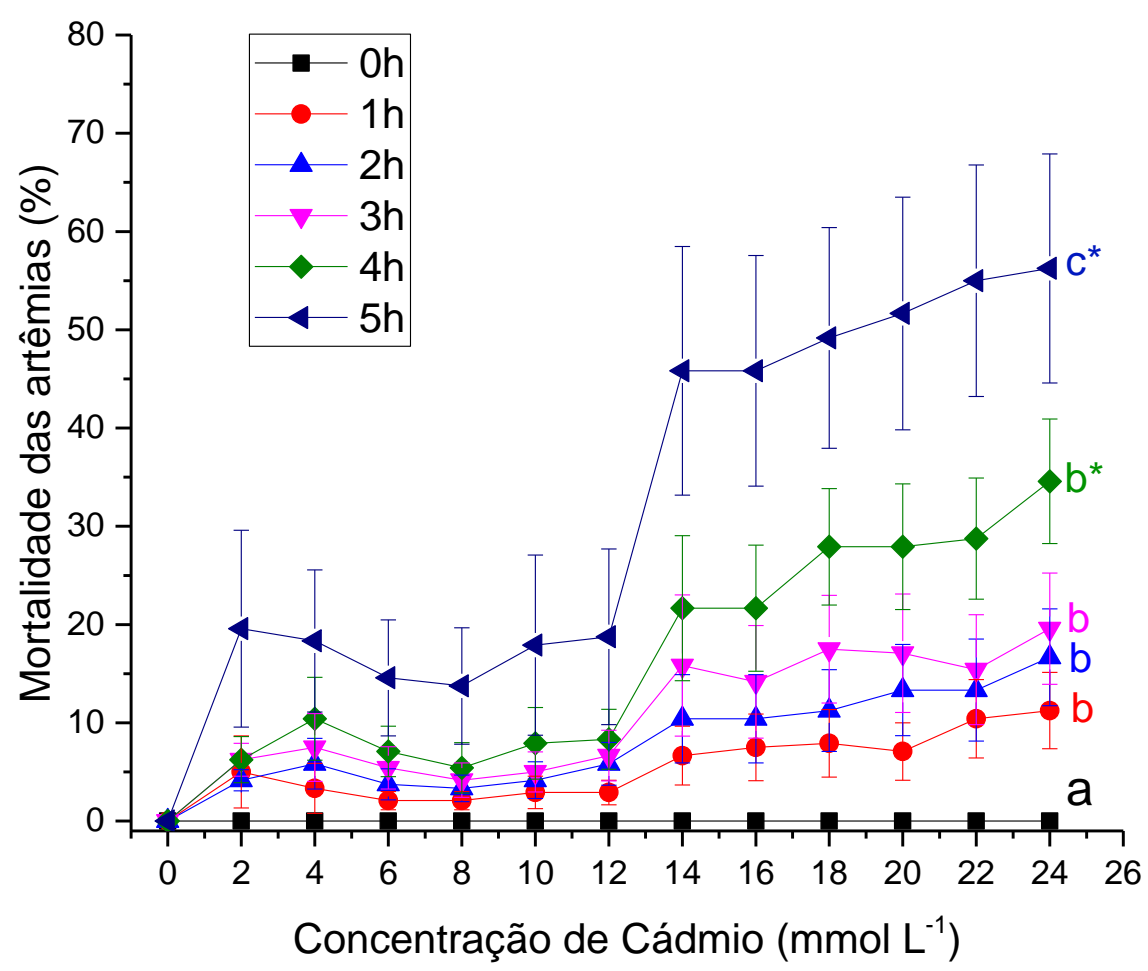

Figura 6. Porcentagem (\%) de morte de artêmias em relação às diferentes concentrações de $\operatorname{CdCl}_{2}(0,2,4,6$, $8,10,12,14,16,18,20,22$ e $24 \mathrm{mmol} \mathrm{L}^{-1}$ ) ao longo de 5 horas de experimento. As diferentes cores representam os diferentes tempos de exposição ao Cd: preto para 0 hora; vermelho para 1 hora; azul para 2 horas; rosa para 3 horas; verde para 4 horas; e azul escuro para 5 horas. As diferentes letras representam as diferenças significativas comparando os grupos experimentais e controle com relação ao tempo de exposição (0 a 5 horas). Os asteriscos representam as diferenças significativas comparando os grupos experimentais e controle com relação às concentrações utilizadas $\left(0\right.$ a $\left.24 \mathrm{mmol} \mathrm{L}^{-1}\right)$.

Nosso formulário de satisfação também apresentou dados importantes com relação ao sexo dos participantes. Os homens estiveram mais presentes no evento, possivelmente devido à localização do mesmo (realizado na UNI). A instituição oferece vários cursos na área das Engenharias, além de Química e Engenharia Ambiental. Os cursos, de maneira geral, são realizados pelo sexo masculino, ocorrendo assim uma grande concentração de homens na universidade. Além disso, a maior parte dos participantes são estudantes de graduação, seguida por estudantes de pós-graduação e outros tipos de profissionais/ocupações. Esse fato sugere que os estudantes de menor faixa etária buscam diversas maneiras de aprendizagem e capacitação, estando próximos de profissionais que atuam nas áreas que pretendem seguir como carreira. Um aspecto que podemos ressaltar é a menor presença de professores e pesquisadores, com maior faixa etária. Esse dado sugere que os professores e pesquisadores poderiam apresentar uma elevada carga horária, ocupados com a docência nos cursos de graduação e pós-graduação, e no desenvolvimento de projetos de pesquisa. Além disso, os profissionais da educação também poderiam pensar que BIOMET abordaria temas mais simples, menos específicos, voltados para os alunos de graduação. Nesse sentido, a inclusão de comunicações orais mais específicas e aprofundadas dentro do tema de Bioinorgânica seria importante para atrair mais alunos de pósgraduação, professores e pesquisadores. Outra problemática se refere à atualização profissional. De acordo com estudos anteriores (Altet 2001), os profissionais da educação apresentam características específicas, que os tornam educadores, como: os conhecimentos, as habilidades e atitudes necessárias para garantir o bom aprendizado. Nesse contexto, a atualização é de extrema importância, pois os conhecimentos são transformados e reformulados ao longo do tempo 
(Madruga 1996). Com a ausência dessa atualização, os profissionais da educação podem se tornar obsoletos, podendo ocasionar lacunas na sua formação teórico-prática, dificultando uma aprendizagem significativa dos discentes (Altet 2001; Lima \& Vasconcelos 2008). Assim, seria de grande importância que a divulgação do evento também ocorresse entre professores e pesquisadores, com o intuito de promover a interação entre participantes e ministrantes do curso para o intercâmbio de experiências e para a atualização e aprendizagem de metodologias práticas que poderiam ser inseridas em aulas de laboratório.

O maior percentual de participação ocorreu nas palestras BIOMET 2017. Esse fato poderia sugerir duas hipóteses: a primeira seria uma maior restrição à participação no minicurso, justamente por ser ministrado em laboratório com capacidade máxima de 30 alunos, o que não comporta todos os participantes do evento. A segunda hipótese seria o maior interesse em palestras e conteúdos ditados de forma oral em detrimento à prática. Contudo, estudos demonstram que as atividades práticas desenvolvidas em todos os contextos educacionais (ensino fundamental, médio e universitário) apresentam maior significância para o aluno (Gomes et al. 2010; Marin et al. 2010), sendo estas, inclusive, mais dinâmicas, onde há a participação do aluno na resolução de questões problemáticas (Freire 1987; Krasilchik 2009). Além disso, os participantes do minicurso apontaram a prática desenvolvida em laboratório como essencial para o melhor entendimento e aprendizado do tema desenvolvido por BIOMET 2017 (dados não mostrados), sendo este dia de minicurso a ligação entre conteúdo teórico e prático. Dessa maneira, acredita-se que a primeira hipótese seria a principal responsável pelo menor número de participantes no minicurso. Assim, para os futuros eventos BIOMET, seria importante ampliar o espaço laboratorial, para que um número maior de alunos possa participar dos experimentos desenvolvidos em laboratório.

Apesar da grande importância em atividades práticas para um aprendizado significativo, a realidade das universidades é diferente da teoria. De todos os participantes, apenas $50 \%$ desses participam de aulas laboratoriais, enquanto que $60 \%$ do total realizam apenas um experimento por aula. Esses dados revelam uma defasagem na ciência e no aprendizado de ciência. As aulas laboratoriais são importantes para o aluno aprender na prática o que é ensinado nas aulas teóricas, relacionando os conteúdos e aplicando os mesmos à realidade onde estão inseridos, aprendendo, assim, de maneira significativa (Valderrama et al. 2016). De acordo com Freire (1987), alunos inseridos em um ensino tradicionalista e conservador, não conseguem relacionar o conteúdo teórico com o contexto e a realidade vivenciada no seu cotidiano. Além disso, o desenvolvimento de habilidades procedimentais (como trabalhar em grupo) são aprimoradas em abordagens metodológicas práticas de ensino. Aulas laboratoriais também são importantes pois estimulam o desenvolvimento e teste de hipóteses para a resolução de situações-problema; o aluno é estimulado a relacionar o conteúdo, a prática e o seu cotidiano, obtendo, assim, um aprendizado amplo e significativo, importante para futuros profissionais na área de ciências (Krasilchik 2009; Gomes et al. 2010; Marin et al. 2010; Valderrama et al. 2016).

Com relação ao evento BIOMET, nota-se que esse é de total importância para a difusão científica como um todo, pois a maioria dos participantes não conheciam eventos de difusão científica nos mesmos moldes de BIOMET 2017. Além disso, o evento apresentou alto grau de satisfação e de qualidade, sendo considerado bom ou muito bom. Estudos anteriores afirmam a importância da difusão científica, justamente porque essa prática de comunicação rompeu com o isolamento da ciência e da vida científica, atenuando o desconhecimento por parte do público em geral (Fayard 1999), ou seja, eventos como BIOMET são essenciais pois visam a popularização da investigação e da tecnologia, instaurando um diálogo entre ciência e sociedade (Fayard 1999). O alto grau de satisfação poderia ocorrer devido à qualidade dos profissionais envolvidos no evento. Todos os profissionais apresentam pós-graduação e especialização nas diversas áreas de Ciências, em instituições renomadas, sendo assim relevantes a experiência e o domínio dos temas propostos pelo evento. Trabalhos mostram que profissionais atualizados e preparados são capazes de desenvolver metodologias diferenciadas de aprendizagem, melhorando o ensino-aprendizagem dos discentes, tornando este significativo (Altet 2001). Com relação aos temas, eles ainda podem ser mais aprofundados e com uma maior diversidade 
experimental, com o intuito de relacionar as diferentes áreas do conhecimento em ciências. A interdisciplinaridade, nesse caso, torna-se importante pois assim os diferentes conteúdos podem ser relacionados entre si, aproximando e contextualizando os diferentes temas explorados (Santos 2008; Luz 2009).

\section{O minicurso}

Os resultados obtidos durante o minicurso, realizado em laboratório, por BIOMET 2017 apontaram um aumento da morte das artêmias ao longo das 5 horas de experimento, principalmente após 1 hora de exposição às mais elevadas concentrações de cádmio (14 a 24 mmol $\left.\mathrm{L}^{-1}\right)$. Além disso, após as 5 horas de experimento, as artêmias apresentaram $65 \%$ de mortalidade. Estudos revelam que, concentrações a partir de $0.054 \mathrm{mmol} \mathrm{L}^{-1}$ já causam efeitos tóxicos detectáveis (Kokkali et al. 2011). Nesse mesmo estudo, a LC $_{50}$ para esses crustáceos foi determinada em $4 \mathrm{mmol} \mathrm{L}^{-1}$. Da mesma forma, as artêmias podem acumular o cádmio em concentrações de $0.54 \mathrm{mmol} \mathrm{L}^{-1}$, ocasionando um acúmulo maior em concentrações de $54 \mathrm{mmol}$ $\mathrm{L}^{-1}$ (Jennings \& Rainbow 1979). Dessa maneira, acredita-se que as concentrações utilizadas para o minicurso foram suficientes para ocasionar a mortalidade das artêmias.

No entanto, durante o minicurso, os participantes apresentaram dificuldades como: 1) contagem das artêmias para serem transferidas para as placas. Por serem nauplios e apresentarem tamanho reduzido, foi difícil a transferência de 24 artêmias para os poços das placas. As artêmias se moviam constantemente, o que dificultava a contagem das mesmas; 2) contagem das artêmias mortas. Apesar das artêmias mortas ficarem aderidas ao fundo da placa, muitos participantes encontraram dificuldades para diferenciar os nauplios vivos dos mortos. $\mathrm{O}$ ideal seria a utilização de um fundo escuro (pois as artêmias são claras) para a melhor contagem, contudo, nem todos os participantes utilizaram desse método, podendo ocorrer erros na contagem; 3) adição das diferentes concentrações do $\mathrm{CdCl}_{2}$. A maioria dos participantes do minicurso nunca haviam manuseado micropipetas e nunca haviam trabalhado com placas de 6 poços, ou com concentrações de metais tóxicos. Apesar do protocolo apresentar passo-a-passo o procedimento de adição das concentrações de cloreto de cádmio, muitos participantes encontraram dificuldades para estabilizar o volume final de cada poço das placas, o que poderia levar a poços com concentrações diferentes (maiores ou menores) em relação ao protocolo estabelecido inicialmente; e 4) cálculo da porcentagem de crustáceos mortos. Possivelmente devido à falha na contagem inicial, muitos participantes não encontraram um aumento progressivo da mortalidade das artêmias.

As dificuldades apresentadas acima poderiam ter interferido nos resultados, sendo plausível a hipótese de que, devido às falhas apresentadas na execução do experimento, ele não foi realizado de maneira satisfatória, contrastando com os dados apresentados na literatura. Essas falhas poderiam ser explicadas devido à falta de aulas práticas nas instituições de ensino de origem dos participantes. Um outro fator que também deve ser levado em consideração é a dificuldade no entendimento do método. Por muitas vezes, os alunos questionaram o protocolo e o desenvolvimento do experimento, encontrando dificuldades no seu entendimento. Estudos ressaltam que, para uma maior eficiência no ensino-aprendizagem, docentes e discentes necessitam de uma boa comunicação. Metodologias e métodos claros, protocolos explicativos e com objetivos bem delineados, facilitam a comunicação e a aprendizagem dos alunos (Freire 1987; Krasilchik 2009).

Os fatores apresentados acima, como as dificuldades na realização experimental, ressaltam ainda mais a importância de eventos como o BIOMET 2017. De acordo com estudos anteriores (Valderrama et al. 2016), práticas experimentais e laboratoriais são necessárias para o desenvolvimento do discente. Esse desenvolvimento ocorre principalmente no que se refere às competências experimentais, onde os discentes adquirem habilidades tanto na execução do experimento em si, quanto na criação de hipóteses e discussão dos dados obtidos (Praia et al. 2002). Dessa maneira, acredita-se que mais eventos de difusão científica, com participação 
experimental, como BIOMET 2017, devem ser desenvolvidos, para uma melhor aprendizagem dos discentes, futuros profissionais na área das Ciências.

\section{Conclusão}

BIOMET 2017 apresentou grande participação masculina, de estudantes de graduação, das áreas de Química e Biologia, com pouca experiência em laboratório, sendo o evento classificado como "bom ou muito bom" pelos participantes. Com relação ao minicurso, a morte das artêmias ocorreu a partir de $10 \mathrm{mmol} \mathrm{L}^{-1}$, após 1 hora de experimento. Estes resultados foram divergentes com a literatura, apresentando certa inconsistência, uma vez que a morte total de artêmias ao final do experimento foi de $65 \%$. Possíveis falhas ocorreram durante o minicurso devido às dificuldades apresentadas durante a execução do experimento, como no entendimento do protocolo, inexperiência na sua execução, e no manuseio de equipamentos. Assim, eventos como BIOMET 2017 são importantes para a difusão científica, pois permite a interação com professores e pesquisadores, e a compreensão de pesquisas científicas desenvolvidas em diferentes instituições. Além disso, o minicurso também contribuiu na aprendizagem e na formação inicial dos alunos, como uma maneira do aluno se aproximar dos equipamentos laboratoriais e da rotina de trabalho de um pesquisador, melhorando a sua formação nas áreas de Ciências.

\section{Agradecimentos}

Facultad de Ciencias da Universidad Nacional de Ingeniería (UNI), pela disponibilização do auditório para as palestras, e do laboratório para o workshop. Aos participantes do evento BIOMET 2017, pela disponibilização dos dados. Ao Biólogo Joseph Fabián Encinas Surco pelo suporte técnico. À Coordenação de Aperfeiçoamento de Pessoal de Nível Superior (CAPES) e à Fundação de Amparo à Pesquisa do Estado de São Paulo (FAPESP) pelo suporte técnico e financeiro.

\section{Referências}

Alberts B. (2009) Redefining science education. Science, 323(5913): 437. https://doi.org/10.1126/science.1170933

Altet M. (2001) As competências do professor profissional: entre conhecimentos, esquemas de ação e adaptação, saber analisar (p. 23-35). In: Paquay L., Altet M., Charlier É. \& Perrenoud P. (Eds). Formando professores profissionais: Quais estratégias. Porto Alegre: Artmed Editora. $232 \mathrm{p}$.

Borochovicius E. \& Tortella C.J.B. (2014) Aprendizagem Baseada em Problemas: um método de ensino-aprendizagem e suas práticas educativas. Ensaio Avaliação e Políticas Públicas em Educação, 22(83): 263-294.

Cotta R.M.M., Silva L.S., Lopes L.L., Gomes K.O., Cotta F.M., Lugarinho R. \& Mitre S.M. (2012) Construção de portfólios coletivos em currículos tradicionais: uma proposta inovadora de ensino-aprendizagem. Ciência \& Saúde Coletiva, 17(3): 787-796.

https://doi.org/10.1590/S1413-81232012000300026

Cyrino E.G. \& Toralles-Pereira M.L. (2004) Trabalhando com estratégias de ensino-aprendizado por descoberta na área da saúde: a problematização e a aprendizagem baseada em problemas. Cadernos de Saúde Pública, 20(3): 780-788. https://doi.org/10.1590/S0102-311X2004000300015

Fagundes T.B. (2016) Os conceitos de professor pesquisador e professor reflexivo: perspectivas do trabalho docente. Revista Brasileira de Educação, 21(65): 281-298.

https://doi.org/10.1590/S1413-24782016216516

Fayard P. (1999) La sorpresa de Copérnico: el conocimiento gira alrededor del público. Alambique Didáctica las Ciencias Experimentales, 6(21): 9-16.

Festas M.I.F. (2015) A aprendizagem contextualizada: análise dos seus fundamentos e práticas pedagógicas. Educação e Pesquisa, 41(3): 713-728. 
https://doi.org/10.1590/S1517-9702201507128518

Freire P. (1987) Pedagogia do oprimido. $17^{\circ}$ edição. Rio de Janeiro: Paz e Terra. 275 p.

Gomes M.P.C., Ribeiro V.M.B., Monteiro D.M., Leher E.M.T. \& Louzada R.C.R. (2010) O uso de metodologias ativas no ensino de graduação nas ciências sociais e da saúde - avaliação dos estudantes. Ciência e Educação, 16(1): 181-198. https://doi.org/10.1590/S1516-73132010000100011

Jennings J.R. \& Rainbow P.S. (1979) Accumulation of cadmium by Artemia salina. Marine Biology, 51(1): 47-53. https://doi.org/10.1007/BF00389030

Kokkali V., Katramados I. \& Newman J.D. (2011) Monitoring the Effect of Metal Ions on the Mobility of Artemia salina Nauplii. Biosensors, 1: 36-45. https://doi.org/10.3390/bios1020036

Krasilchik M. (2009) Biologia - ensino prático (p. 249-258). In: Caldeira A.M.A. \& Araújo E.S.N.N. (Eds). Introdução à Didática da Biologia. São Paulo: Educação para a Ciência - Escrituras. 304 p.

Lima K.E.C. \& Vasconcelos S.D. (2008) Science teachers from public schools in Recife and their perspective on continuing education. Ciência \& Educação, 14(2): 347-364. https://doi.org/10.1590/S1516-73132008000200012

Lima J.M., Silva C.A., Rosa M.B., Santos J.B., Oliveira T.G. \& Silva M.B. (2009) Prospecção fitoquímica de Sonchus oleraceus e sua toxicidade sobre o microcrustáceo Artemia salina. Planta Daninha, 27(1): 7-11.

Luz M.T. (2009) Complexidade do campo da Saúde Coletiva: multidisciplinaridade, interdisciplinaridade, e transdisciplinaridade de saberes e práticas - análise sócio-histórica de uma trajetória paradigmática. Saúde e Sociedade, 18: 304-311. https://doi.org/10.1590/S0104-12902009000200013

Madruga A. (1996) Aprendizagem pela descoberta frente à aprendizagem pela recepção: a teoria da aprendizagem verbal significativa (p. 68-78). In: Coll C., Palácios J. \& Marchesi A. (Eds). Desenvolvimento psicológico e educação. Porto Alegre: Artes Médicas. 381 p.

Marin M.J.S., Gomes R., Marvulo M.M.L., Primo E.M., Barbosa P.M.K. \& Druzian S. (2010) Pósgraduação multiprofissional em saúde: resultados de experiências utilizando metodologias ativas. Interface - Comunicação, Saúde, Educação, 14: 331-344. https://doi.org/10.1590/S1414-32832010000200008

Ørngreen R. \& Levinsen K. (2017) Workshops as a Research Methodology. The Electronic Journal of e-Learning, 15: 70.

Praia J., Cachapuz A. \& Gil-Pérez D. (2002) A hipótese e a experiência científica em educação em ciência: contributos para uma reorientação epistemológica. Ciência \& Educação, 8(2): 253262. https://doi.org/10.1590/S1516-73132002000200009

Rohlf F.J. \& Sokal R.R. (1969) Statistical tables. 5 illus. Folkestone: W.H Freeman and Company Ltd. 253 p.

Santos A. (2008) Complexidade e transdisciplinaridade em educação. Revista Brasileira de Educação, 13(37): 71-83. https://doi.org/10.1590/S1413-24782008000100007

Scarpa D.L. (2015) O papel da argumentação no ensino de ciências: lições de um workshop. Ensino e Pesquisa em Educação em Ciências, 17: 15-30. https://doi.org/10.1590/1983-2117201517s02

Valadares J. (2011) The meaningful learning theory as a constructivist theory. Meaningful Learning Review, 1: 36-57.

Valderrama L., Belinato Paiva V., Março P.H. \& Valderrama P. (2016) Proposta experimental didática para o ensino de análise de componentes principais. Química Nova, 39(2): 245-249. https://doi.org/10.5935/0100-4042.20150166

Wieman C. (2007) Why Not Try a Scientific Approach to Science Education? Change: The Magazine of Higher Learning, 39(5): 9-15. https://doi.org/10.3200/CHNG.39.5.9-15

Wieman C. \& Gilbert S. (2015) Taking a Scientific Approach to Science Education, Part II Changing Teaching. Microbe, 10(5): 203-207. 